TAIWANESE JOURNAL OF MATHEMATICS

Vol. 7, No. 4, pp. 657-664, December 2003

This paper is available online at http://www.math.nthu.edu.tw/tjm/

\title{
ON $P_{4}$-DECOMPOSITION OF GRAPHS
}

\author{
C. Sunil Kumar
}

\begin{abstract}
A graph $G$ is decomposable into subgraphs $G_{1}, G_{2}, \ldots, G_{n}$ of $G$ if no $G_{i}(i=1,2, \ldots, n)$ has isolated vertices and the edge set $E(G)$ can be partitioned into the subsets $E\left(G_{1}\right), E\left(G_{2}\right), \ldots, E\left(G_{n}\right)$. If $G_{i} \cong P_{4}$ for all $i$, then $G$ is called $P_{4}$-decomposable. In this paper, we show the $P_{4}$-decomposability of some classes of graphs, and prove in particular that a complete $r$-partite graph is $P_{4}$-decomposable if and only if its size is a multiple of 3. We also give an example of a 2-connected graph of size $3 k$ which is not $P_{4}$-decomposable, disproving a conjecture of Chartrand.
\end{abstract}

\section{INTRODUCTION}

In this paper we only consider simple graphs. A graph $G$ is said to be $H$ decomposable, denoted by $H \mid G$, if $E(G)$ can be partitioned into subgraphs such that each subgraph is isomorphic to $H$. Such a factorization is called an isomorphic factorization. The concept of isomorphic factorization was studied by F. Harary et al. [4]. In this paper we consider a conjecture of Chartrand et al. [3] that a 2-connected graph of order $p \geq 4$ and size $q \equiv 0(\bmod 3)$ is $P_{4}$-decomposable. We prove the conjecture for certain 2-connected graphs. We also show by an example that it is not true in general.

We follow standard notations in graph theory. The cardinality of the vertex set of a graph $G$, the order of $G$ is denoted by $p(G)$; and the cardinality of the edge set of $G$, the size of $G$ is denoted by $q(G)$.

Theorem 1. $K_{m, n}$ is $P_{4}$-decomposable if and only if $m \geq 2, n \geq 2$ and $m n \equiv 0(\bmod 3)$.

Received September 1, 2001; revised May 30, 2002.

Communicated by G. J. Chang.

2000 Mathematics Subject Classification: 05C70.

Key words and phrases: Edge decomposition. 
Proof. As the conditions are clearly necessary, we only need to prove the sufficiency. Without loss of generality, we may assume that $m=3 r$. Write $n=$ $2 s+3 t$ with $s \geq 0$ and $t \geq 0$. Then $K_{m, n}$ can be decomposed into $r s$ copies of $K_{2,3}$ and $r t$ copies of $K_{3,3}$. It is easily verified that $K_{2,3}$ and $K_{3,3}$ are $P_{4}$-decomposable. Hence $K_{m, n}$ is $P_{4}$-decomposable.

Theorem 2. If $G_{1}, G_{2}$ and $K_{m_{1}, m_{2}}$ are $H$-decomposable, where $m_{1}=p\left(G_{1}\right)$ and $m_{2}=p\left(G_{2}\right)$, then $G_{1}+G_{2}$ is H-decomposable.

Proof. As $E\left(G_{1}+G_{2}\right)$ is equal to the edge-disjoint union $E\left(G_{1}\right) \cup E\left(G_{2}\right) \cup$ $E\left(K_{m_{1}, m_{2}}\right)$, we have that $G_{1}+G_{2}$ is $H$-decomposable.

Theorem 3. If $G_{1}$ and $G_{2}$ are $P_{4}$-decomposable graphs and $p\left(G_{1}\right)$ or $p\left(G_{2}\right)$ is a multiple of 3 , then $G_{1}+G_{2}$ is $P_{4}$-decomposable.

Proof. Let $p\left(G_{1}\right)=m$ and $p\left(G_{2}\right)=n$. Then $K_{m, n}$ is $P_{4}$-decomposable by Theorem 1. By Theorem 2, $G_{1}+G_{2}$ is $P_{4}$-decomposable.

Theorem 4. If $G_{1}, G_{2}, \ldots, G_{n}$ are $P_{4}$-decomposable graphs and $p\left(G_{i}\right) \equiv 0$ $(\bmod 3)$ for $i=1,2, \ldots, n$, then $G_{1}+G_{2}+\ldots+G_{n}$ is $P_{4}$-decomposable.

Proof. The theorem holds for the case $n=2$ by Theorem 3. The general case follows from an induction on $n$, as $G_{1}+G_{2}+\ldots+G_{n} \cong\left(G_{1}+G_{2}\right)+\ldots+G_{n}$.

Lemma 5. If $K_{r}$ and $K_{r, r}$ are $H$-decomposable, then $K_{n r}$ is $H$-decomposable for any positive integer $n$.

Proof. We prove the lemma by induction on $n$. When $n=1, K_{r}$ is $H$ decomposable by the assumption. Assume the lemma is true for $n=m-1 \geq 1$. We prove that the lemma is true for $n=m$. Notice that $K_{m r}=K_{(m-1) r+r}$ and $E\left(K_{(m-1) r+r}\right)=E\left(K_{(m-1) r}\right) \bigcup E\left(K_{r}\right) \bigcup E\left(K_{(m-1) r, r}\right)$. By the induction hypothesis, $K_{(m-1) r}$ is H-decomposable. As $K_{(m-1) r, r}$ can be decomposed into $m-1$ copies of $K_{r, r}$, we have that $K_{(m-1) r, r}$ is $H$-decomposable. Thus $K_{m r}$ is $H$-decomposable. These prove the lemma.

Using Theorems 1 and 2 and Lemma 5, we have the following propositions.

Proposition 6. When $n \geq 2$ and $m \geq 1, K_{3 n}+P_{3 m+1}$ is $P_{4}$-decomposable.

Proposition 7. When $n \geq 2$ and $m \geq 2, K_{3 n}+C_{3 m}$ is $P_{4}$-decomposable.

Proposition 8. When $n \geq 2$ and $m \geq 2, C_{3 n}+C_{3 m}$ is $P_{4}$-decomposable. 
Theorem 9. $K_{n}$ is $P_{4}$-decomposable if and only if $n>3$ and $n \not \equiv 2(\bmod 3)$.

Proof. Clearly $K_{n}$ is not $P_{4}$-decomposable for $n \leq 3$. Also, if $n \equiv 2(\bmod 3)$, then $q\left(K_{n}\right)=\frac{n(n-1)}{2}$ is not divisible by 3 and hence $K_{n}$ is not $P_{4}$-decomposable.

It can be easily verified that $K_{4}$ is $P_{4}$-decomposable. So, let $n$ be an integer such that $n \not \equiv 2(\bmod 3)$ and $n \geq 6$.

Case 1. $n \equiv 0(\bmod 3)$.

When $n$ is odd, $K_{n}$ is decomposable into $\frac{n-1}{2}$ Hamiltonian cycles each of which is $P_{4}$-decomposable. It is also easily verified that $K_{6}$ is $P_{4}$-decomposable. Notice that $E\left(K_{6 r}\right)=E\left(K_{6(r-1)}\right) \bigcup E\left(K_{6}\right) \bigcup E\left(K_{6(r-1), 6}\right)$. It follows from an induction on $r$ that $K_{6 r}$ is $P_{4}$-decomposable.

Case 2. $n \equiv 1(\bmod 3)$, say $n=3 k+1$.

We first show that $K_{7}$ is $P_{4}$-decomposable. Let the vertices of $K_{7}$ be $v_{0}, v_{1}, v_{2}$, $v_{3}, v_{4}, v_{5}, v_{6}$. Then $K_{7}$ can be decomposed into 3 Hamiltonian cycles as follows:

$$
\begin{aligned}
& C^{1}: v_{0}, v_{1}, v_{2}, v_{6}, v_{3}, v_{5}, v_{4}, v_{0} ; \\
& C^{2}: v_{0}, v_{2}, v_{3}, v_{1}, v_{4}, v_{6}, v_{5}, v_{0} ; \\
& C^{3}: v_{0}, v_{3}, v_{4}, v_{2}, v_{5}, v_{1}, v_{6}, v_{0} .
\end{aligned}
$$

The edges $\left\{v_{4}, v_{0}\right\}$ from $C^{1},\left\{v_{0}, v_{2}\right\}$ from $C^{2}$ and $\left\{v_{2}, v_{5}\right\}$ from $C^{3}$ form a path $P_{4}$. The other edges of $C^{1}, C^{2}, C^{3}$ form 2 paths $P_{4}$ each. Hence $K_{7}$ is $P_{4}$ decomposable. Notice that $E\left(K_{3 k+1}\right)=E\left(K_{3(k-1)}\right) \cup E\left(K_{4}\right) \cup E\left(K_{3(k-1), 4}\right)$ and each of the graphs on the right is $P_{4}$-decomposable if $k \geq 3$. Hence $K_{3 k+1}$ is $P_{4^{-}}$ decomposable for all integers $k \geq 1$.

These complete the proof of the theorem.

Theorem 10. If $n \equiv 2(\bmod 3)$ and $n>4$, then $K_{n}-e$ is $P_{4}$-decomposable.

Proof. It can be easily verified that $K_{5}-e$ is $P_{4}$-decomposable. For $n>5$, we have $E\left(K_{n}-e\right)=E\left(K_{2, n-2}\right) \bigcup E\left(K_{n-2}\right)$. Since $n-2 \equiv 0(\bmod 3), K_{2, n-2}$ is $P_{4}$-decomposable by Theorem 1 , and $K_{n-2}$ is $P_{4}$-decomposable by Theorem 9 . The theorem then follows.

Proposition 11. If $n \equiv 0(\bmod 3)$, then $K_{2 n}-F$ is $P_{4}$-decomposable, where $F$ is a 1-factor of $K_{2 n}$.

Proof. The proposition follows from the fact that $K_{2 n}-F$ can be decomposed into $n-1$ Hamiltonian cycles, each of which is $P_{4}$-decomposable. 
Proposition 12. A wheel $W_{n}$ is $P_{4}$-decomposable if and only if $n \equiv 0(\bmod$ $3)$.

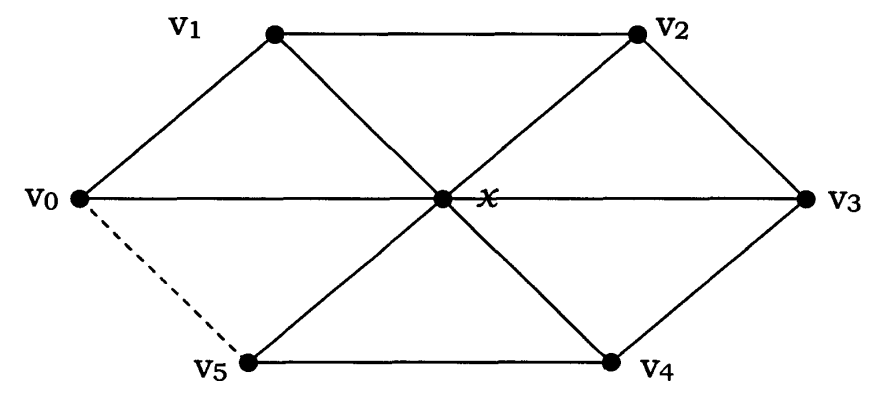

Proof. The condition is clearly necessary.

Conversely, suppose that $n \equiv 0(\bmod 3)$. It is clear that $W_{n}=C_{n}+K_{1}$. Let $C_{n}$ be the cycle $v_{0}, v_{1}, v_{2}, \ldots, v_{n-1}, v_{0}$ and $K_{1}=x$. Notice that $q\left(W_{n}\right)=2 n \equiv 0$ $(\bmod 3)$. It is a routine to check that $E\left(W_{n}\right)$ can be decomposed into $\frac{2 n}{3} P_{4}$ 's of the form $x v_{0+3 i}, v_{1+3 i}, v_{2+3 i}$, and $v_{1+3 i}, x v_{2+3 i}, v_{3+3 i}$, where $0 \leq i=\leq \frac{n}{3}-1$ and $v_{n}=v_{0}$.

Theorem 13. Let $G$ be a complete tripartite graph $K_{m_{1}, m_{2}, m_{3}}$. Then $G$ is $P_{4}$-decomposable if and only if $q(G) \equiv 0(\bmod 3)$ and $q(G)>3$.

Proof. We only need to prove the sufficiency. Since $q(G) \equiv 0(\bmod 3)$, there are three possibilities:

1. $m_{i} \equiv 1(\bmod 3)$ for all $i$;

2. $m_{i} \equiv 2(\bmod 3)$ for all $i$;

3. $m_{i} \equiv 0(\bmod 3)$ for at least two $i$.

Case 1. $m_{i} \equiv 1(\bmod 3)$ for $i=1,2,3$.

Let $m_{1}=3 a+1, m_{2}=3 b+1$ and $m_{3}=3 c+1$. Notice that

$$
\begin{aligned}
& E\left(K_{3 a+1,3 b+1,3 c+1}\right)=E\left(K_{3 a, 3 b+1+3 c+1}\right) \cup E\left(K_{1,3 b+1,3 c+1}\right), \\
& E\left(K_{1,3 b+1,3 c+1}\right)=E\left(K_{3 b, 1+3 c+1}\right) \bigcup E\left(K_{1,1,3 c+1}\right), \\
& E\left(K_{1,1,3 c+1}\right)=E\left(K_{3(c-1), 2}\right) \bigcup E\left(K_{1,1,4}\right) .
\end{aligned}
$$

By Theorem 1, $K_{3 a, 3 b+1+3 c+1}, K_{3 b, 1+3 c+1}$ and $K_{3(c-1), 2}$ are $P_{4}$-decomposable. [If $c=1, K_{3(c-1), 2}$ is a null graph.]

A $P_{4}$-decomposition of $K_{1,1,4}$ is shown below. 


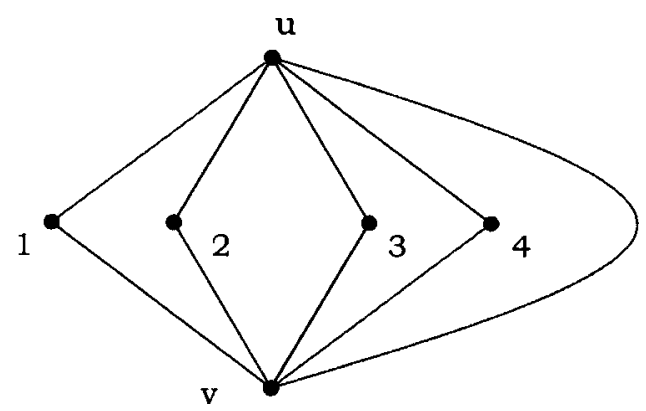

$(1, u, v, 4),(1, v, 2, u),(v, 3, u, 4)$ is a $P_{4}$-decomposition of $K_{1,1,4}$. Thus $K_{m_{1}, m_{2}, m_{3}}$ is $P_{4}$-decomposable when $m_{i} \equiv 1(\bmod 3)$ for $i=1,2,3$.

Case 2. $m_{i} \equiv 2(\bmod 3)$ for $i=1,2,3$.

Let $m_{1}=3 a+2, m_{2}=3 b+2$ and $m_{3}=3 c+2$. Notice that

$$
\begin{aligned}
& E\left(K_{3 a+2,3 b+2,3 c+2}\right)=E\left(K_{3 a, 3 b+2+3 c+2}\right) \bigcup E\left(K_{2,3 b+2,3 c+2}\right), \\
& E\left(K_{2,3 b+2,3 c+2}\right)=E\left(K_{3 b, 2+3 c+2}\right) \bigcup E\left(K_{2,2,3 c+2}\right), \\
& E\left(K_{2,2,3 c+2}\right)=E\left(K_{3 c, 2+2}\right) \bigcup E\left(K_{2,2,2}\right) .
\end{aligned}
$$

By Theorem $1, K_{3 a, 3 b+2+3 c+2}, K_{3 b, 2+3 c+2}$ and $K_{3 c, 4}$ are $P_{4}$-decomposable. A $P_{4}$-decomposition of $K_{2,2,2}$ is shown below.

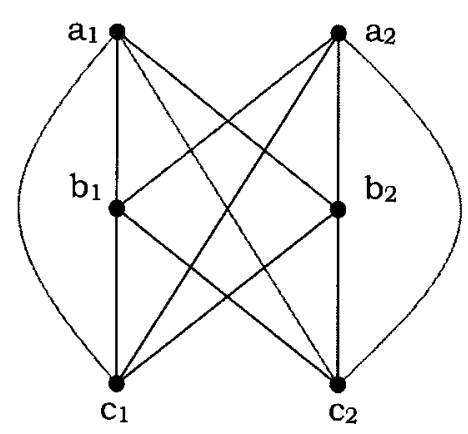

$a_{1} b_{2} a_{2} b_{1} ; b_{1} c_{2} b_{2} c_{1} ; a_{2} c_{2} a_{1} c_{1} ; a_{1} b_{1} c_{1} a_{2}$ is a $P_{4}$-decomposition of $K_{2,2,2}$.

Thus $K_{m_{1}, m_{2}, m_{3}}$ is $P_{4}$-decomposable when $m_{i} \equiv 2(\bmod 3)$ for $i=1,2,3$.

Case 3. $m_{i} \equiv 0(\bmod 3)$ for at least two $i$.

Subcase 3.1. $m_{1}, m_{2} \equiv 0(\bmod 3)$ and $m_{3} \neq 1$. 
Notice that $E\left(K_{m_{1}, m_{2}, m_{3}}\right)=E\left(K_{m_{1}, m_{2}+m_{3}}\right) \bigcup E\left(K_{m_{2}, m_{3}}\right) . K_{m_{1}, m_{2}+m_{3}}$ and $K_{m_{2}, m_{3}}$ are complete bipartite graphs of size a multiple of 3 and hence they are $P_{4}$-decomposable. Thus $K_{m_{1}, m_{2}, m_{3}}$ is $P_{4}$-decomposable.

Subcase 3.2. $m_{1}, m_{2} \equiv 0(\bmod 3)$ and $m_{3}=1$.

Let $m_{1}=3 a$ and $m_{2}=3 b$. Notice that

$$
\begin{aligned}
& E\left(K_{1,3 a, 3 b}\right)=E\left(K_{3(a-1), 1+3 b}\right) \bigcup E\left(K_{1,3,3 b}\right), \\
& E\left(K_{1,3,3 b}\right)=E\left(K_{3(b-1), 1+3}\right) \bigcup E\left(K_{1,3,3}\right) .
\end{aligned}
$$

By Theorem1, $K_{3(a-1), 1+3 b}$ and $K_{3(b-1), 1+3}$ are $P_{4}$-decomposable. A $P_{4}$-decomposition of $K_{1,3,3}$ is shown below.

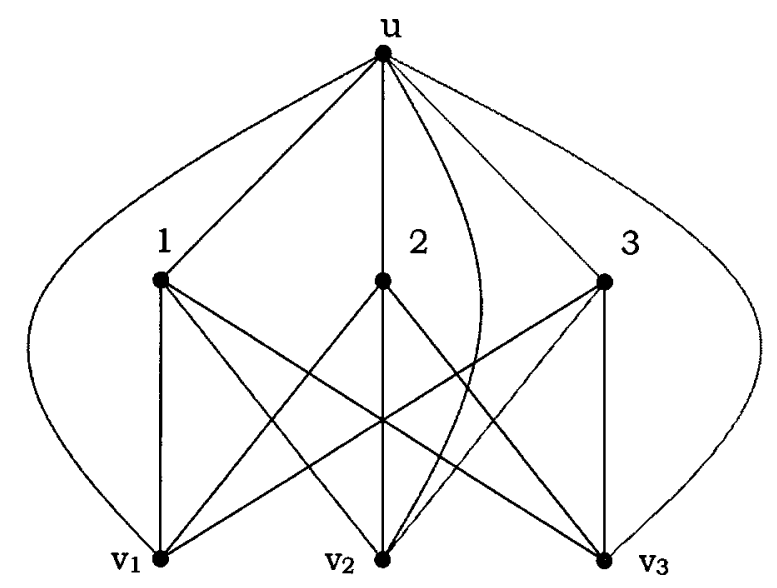

$K_{1,3,3}$ can be decomposed into 3 paths $P_{4}: v_{1}, u, v_{2}, 1 ; 1, u, 2, v_{2} ; v_{2}, 3, u, v_{3}$; and the remaining edges form $K_{3,2}$ which is $P_{4}$-decomposable.

Thus $K_{m_{1}, m_{2}, m_{3}}$ is $P_{4}$-decomposable.

Observation 14. Let $G=K_{3 n_{1}+r_{1}, 3 n_{2}+r_{2}, \ldots, 3 n_{k}+r_{k}}$, where $r_{1}, r_{2}, \ldots, r_{k}$ are positive integers and $n_{1}, n_{2}, \ldots, n_{k}$ are non-negative integers. Then $E(G)=$ $E\left(K_{3 n_{1}, 3\left(n_{2}+\ldots+n_{k}\right)+\left(r_{2}+\ldots+r_{k}\right)}\right) \bigcup E\left(K_{r_{1}, 3 n_{2}+r_{2}, \ldots, 3 n_{k}+r_{k}}\right)$. A similar argument as in Case 2 of Theorem 13 shows that $G$ is $P_{4}$-decomposable if $K_{r_{1}, r_{2}, \ldots, r_{k}}$ is $P_{4^{-}}$ decomposable. This observation is repeatedly used in the proof of the next theorem.

Theorem 15. Let $G$ be the graph $K_{m_{1}, m_{2}, \ldots, m_{r}}$ with $r \geq 4$. Then $G$ is $P_{4}$-decomposable if and only if $q(G) \equiv 0(\bmod 3)$.

Proof. It is enough to prove the sufficiency of the condition. We prove the result by induction on $q=q(G)$. When $q=6, G=K_{4}$ is $P_{4}$-decomposable. When $q=9, G=K_{2,1,1,1}$ is also $P_{4}$-decomposable. 
Case 1. At least one $m_{i} \equiv 0(\bmod 3)$.

Let $m_{1} \equiv 0(\bmod 3)$. Then $E(G)=E\left(K_{m_{1}, m_{2}+m_{3}+\ldots+m_{r}}\right) \bigcup E\left(K_{m_{2}, m_{3}, \ldots, m_{r}}\right)$. Let $G_{1}$ be the graph $K_{m_{1}, m_{2}+m_{3}+\ldots+m_{r}}$ and $G_{2}$ be the graph $K_{m_{2}, m_{3}, \ldots, m_{r}} . G_{1}$ is $P_{4}$-decomposable by Theorem 1 . If $r>4, G_{2}$ is $P_{4}$-decomposable by the induction hypothesis. If $r=4$, except in the case $m_{2}=m_{3}=m_{4}=1$, it follows by Theorem 13 that $G_{2}$ is $P_{4}$-decomposable. So, let us assume $r=4$, $m_{2}=m_{3}=m_{4}=1$. It is easily verified that $K_{3,1,1,1}$ is $P_{4}$-decomposable. If $n>1, E\left(K_{3 n, 1,1,1}\right)=E\left(K_{3,1,1,1}\right) \bigcup E\left(K_{3(n-1), 3}\right)$ and it can be proved by induction on $n$ that $K_{3 n, 1,1,1}$ is $P_{4}$-decomposable for all $n \geq 4$. Thus $G_{2}$ and hence $G$ is $P_{4}$-decomposable in this case.

Case 2. At least three of the $m_{i}$ 's $\equiv 2(\bmod 3)$.

Let $m_{1} \equiv m_{2} \equiv m_{3}=2(\bmod 3)$. Hence $m_{1}+m_{2}+m_{3} \equiv 0(\bmod 3)$ and $E(G)=E\left(K_{m_{1}, m_{2}, m_{3}}\right) \bigcup E\left(K_{m_{1}+m_{2}+m_{3}, m_{4}, m_{5}, \ldots, m_{r}}\right)$. By Theorem 13, the first graph on the right side is $P_{4}$-decomposable. Again, as in Case 1 , if either $r \geq 5$, or if $r=4, m_{4}>1$, the second graph on the right side is $P_{4}$-decomposable. Hence we may assume $r=4$ and $m_{4}=1$. $K_{2,2,2,1}$ is easily verified to be $P_{4}$-decomposable. Hence, by Observation $14, G$ is $P_{4}$-decomposable.

Case 3. Exactly two $m_{i}^{\prime} \mathrm{s}=2(\bmod 3)$.

In this case, it can be verified that $q(G) \not \equiv 0(\bmod 3)$.

Case 4. Exactly one $m_{i} \equiv 2(\bmod 3)$.

In this case $r \equiv 1(\bmod 3) . \quad K_{2,1,1, \ldots, 1} \cong K_{r+1}-e$ is $P_{4}$-decomposable when $r+1 \equiv 2(\bmod 3)$, by Theorem 10 . Hence by Observation $14, G$ is $P_{4-}$ decomposable.

Case 5. No $m_{i} \equiv 2(\bmod 3)$. That is, all $m_{i}{ }^{\prime} s \equiv 1(\bmod 3)$.

In this case $r \equiv 0$ or $1(\bmod 3)$. $K_{1,1,1, \ldots, 1}$ is $P_{4}$-decomposable, by Theorem 9 . Hence by Observation $14, G$ is $P_{4}$-decomposable.

These complete the proof of the theorem.

Conjecture (Chartrand et al. [3]). If $G$ is a 2-connected graph of order $p \geq 4$ and size $q \equiv 0(\bmod 3)$, then $G$ is $P_{4}$-decomposable.

The following example shows that this conjecture is not true. 


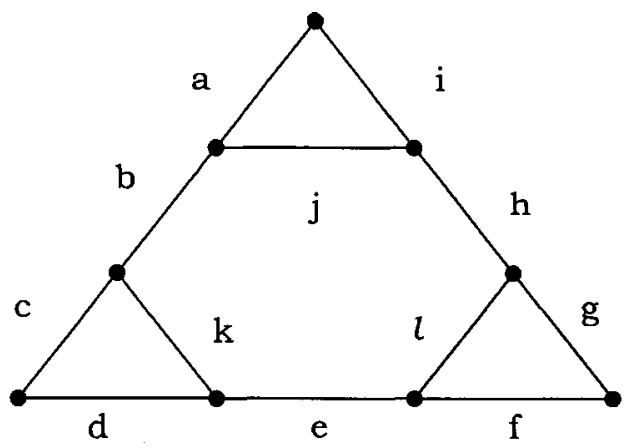

It is easy to see that every $P_{4}$ must contain at least one of the edges $\mathrm{b}$, e and $\mathrm{h}$. Since the graph is of size 12 , it cannot be decomposed into 4 edge-disjoint paths $P_{4}$.

Conjecture. Every 3-connected graph of size $q \equiv 0(\bmod 3)$, is $P_{4}$-decomposable.

\section{ACKNOWLEDGEMENTS}

The author is grateful to Professor M. I. Jinnah, Department of Mathematics, University of Kerala, for his comments and suggestions in every stage of this paper. The author is also pleased to acknowledge several valuable suggestions and many helpful comments made by the referee, in the preparation of the final manuscript. The author is supported by UGC vide their grant No. KLKE 027

\section{REFERENCES}

1. G. J. Chang, Algorithmic aspects of linear $k$-arboricity, Taiwanese J. Math. 3 (1999), 73-81.

2. G. Chartrand and L. Lesniak, Graphs and Digraphs, Second edition, Wordsworth \& Brookes/Cole Monetary (1986).

3. G. Chartrand, F. Saba, and C. M. Mynhardt, Prime graphs, prime-connected graphs and prime divisors of graphs, Utilitas Math. 46 (1994), 179-191.

4. F. Harary, R. W. Robinson, and N. C. Wormald, Isomorphic factorization I: complete graphs, Trans. Amer. Math. Soc. 242 (1978), 243-260.

5. H.-G. Yeh and G. J. Chang, The path-partition problem in bipartite distance-hereditary graphs, Taiwanese J. Math. 2 (1998), 353-360.

C. Sunil Kumar

Department of Mathematics, University of Kerala

Kariavattom, Trivandrum, South India

E-mail: sunic@rediffmail.com 